\section{Lesões visíveis na hanseníase: o papel do cirurgião-dentista na suspeita de casos novos}

\section{Visible lesions in leprosy: The role of dentists in the identification of new cases}

\section{Denise da Costa Boamorte Cortela'}

\section{Eliane Ignotti ${ }^{2}$}

${ }^{1}$ Instituto de Saúde Coletiva (ISC) da Universidade Federal de Mato Grosso (UFMT), Cuiabá

${ }^{2}$ Instituto de Saúde Coletiva (ISC) da Universidade Federal de Mato Grosso (UFMT); Departamento de Enfermagem da Universidade Estadual de Mato Grosso (UNEMAT), Cáceres

Os autores informam não haver nenhum interesse comercial ou associativo que represente conflito de interesse em conexão com o trabalho submetido.

Correspondência: Denise da Costa Boamorte Cortela. Rua das Maravilhas, n¹590, Bairro Cavalhada, Cáceres, MT CEP 78.200-000. E-mail: denisecortela@hotmail.com

\section{Resumo}

Introdução: A hanseníase permanece como problema de saúde pública em alguns países. No Brasil, essa enfermidade demanda a priorização das ações de saúde focadas em alguns Estados e municípios. Objetivo: Analisar as principais características epidemiológicas dos portadores de hanseníase com ênfase na área de localização das lesões. Método: Estudo transversal de indivíduos com hanseníase diagnosticados entre 2001 a 2006, em Cáceres-MT. Foi definida como variável dependente a área de localização das lesões, categorizada em não-visível e visível, que inclui face, pavilhão auricular ou membros superiores. Utilizou-se ANOVA e regressão múltipla logística, com intervalo de confiança de 95\%. Resultados: 206 (33,8 \%) indivíduos apresentaram lesões em áreas visíveis; não foi verificada diferença estatisticamente significante entre os grupos de idade ou sexo com área de localização das lesões. A probabilidade de um paciente multibacilar manifestar lesões em áreas visíveis foi 3 vezes aquela observada entre os pacientes paucibacilares $\left(\mathrm{OR}_{\text {ajust }}=3,21\right.$; IC 95\%: 2,13-4,86). Tal probabilidade entre os pacientes com incapacidade física localizada na região ocular e dos membros superiores foi 5 vezes aquela observada entre os pacientes com incapacidade física localizada em outra região do corpo $\left(\mathrm{OR}_{\text {ajust }}\right.$ = 5,10; IC 95\%: 1,41 - 18,39). Conclusão: A probabilidade de um indivíduo portador de hanseníase apresentar lesões em áreas visíveis independe de sexo e idade e essas se manifestam principalmente nos braços. Entre os indivíduos multibacilares, tal probabilidade foi maior que aquela observada entre os indivíduos paucibacilares, principalmente quando havia presença de incapacidade física localizada na região ocular e nos membros superiores.

Palavras-chave: Hanseníase. Saúde Bucal. Odontologia em Saúde Pública. Epidemiologia. Prevenção de Doenças. Atenção à Saúde. 


\section{Abstract}

Introduction: Leprosy remains a public health problem in some countries and in Brazil it demands health actions focused on some states and municipalities. Objective: To analyze the epidemiologic characteristics of leprosy cases with emphasis on the site of skin lesions. Methods: Cross-sectional study of clinical records of leprosy cases diagnosed during 2001 to 2006 in the municipality of Cáceres-MT. The dependent variable was categorized in visible and not-visible areas including face, pinna, and upper limbs. The analysis was made using ANOVA and logistic regression with a 95\% confidence interval. Results: 206 individuals (33.8\%) presented visible lesions. The probability of a multibacillary patient presenting a visible lesion was three times greater than that observed among paucibacilary patients, regardless of gender and age group (OR $=3.21$; IC 95\%: 2.13 - 4.86). Among multibacillary cases with physical disability located in the ocular region and upper limbs, this probability was five times greater than that observed among those with disability located in another regions of the body $\left(\mathrm{OR}_{\text {ajust }}=5.10\right.$; CI $95 \%$ : 1.41 - 18.39). Conclusion: The likelihood of a Hansen's disease patient presenting lesions in visible areas is independent from gender and age, and they occur mainly in arms. However, among the multibacilary individuals such probability is greater than the one observed among paucibacillary individuals, especially when there is a physical disability located in the ocular region and in the upper limbs.

Keywords: Leprosy. Oral Health. Public Health Dentistry. Epidemiology. Disease Prevention. Health Care.

\section{Introdução}

Entre as ações para eliminação e controle da hanseníase como problema de saúde pública está o aumento da oferta de serviços prestados por profissionais da rede básica com integração das atividades de detecção precoce dos casos $^{1}$. Segundo Ignotti ${ }^{2}$, a eficiência do programa depende da construção de um trabalho em equipe e de estratégias voltadas para a detecção precoce de casos, uma vez que o diagnóstico tardio pode vir a comprometer as metas de eliminação pela manutenção das fontes de contágio e, como conseqüência, a persistência da transmissibilidade ${ }^{3}$.

Deve-se suspeitar de um caso de hanseníase quando uma pessoa apresenta lesões ou áreas na pele (hipopigmentadas ou hiperpigmentadas), com alteração de sensibilidade, dormência ou formigamento das mãos e/ou pés, infiltração na face ou lóbulos da orelha ${ }^{4}$.

Aproximadamente $70 \%$ dos pacientes de hanseníase podem ser diagnosticados pela presença de lesão de pele com perda de sensibilidade ${ }^{5}$. No Brasil, um caso de hanseníase é definido quando há presença de lesão(ões) na pele com perda de sensibilidade bem definida ${ }^{6}$. A simplificação dos métodos de diagnóstico decorrente da presença de alguns sinais cardinais e devido à predileção pela pele conferir características peculiares a esta moléstia, possibilita o envolvimento de todos os profissionais de saúde visando a identificação de casos da doença.

Ainda que o desenvolvimento das lesões possa acometer qualquer região do corpo, as áreas descritas como mais acometidas correspondem à face, pavilhão auricular, membros superiores e inferiores ${ }^{7-12}$.

Segundo Almeida et al. ${ }^{13}$, no que se refere à atividade odontológica, o exame clínico deve se estender além da cavidade bucal, permitindo não só o reconhecimento de sinais e sintomas oriundos de alterações do complexo buco-maxilo-facial, mas também a obtenção de informações sobre a saúde geral do paciente. Esta atitude contempla 
os deveres fundamentais estabelecidos pelo Código de Ética Odontológica, em que cabe ao profissional da área odontológica "promover a saúde coletiva no desempenho de suas funções, cargos e cidadania, independente de exercer a profissão no setor público ou privado" ${ }^{14}$. Assim, em municípios endêmicos, indivíduos com lesões de pele em áreas visíveis poderiam ser identificados como casos suspeitos de hanseníase pelo cirurgião-dentista durante um exame clínico de rotina, ainda que estes dependam de exame médico para confirmação diagnóstica.

Considerando a possibilidade de contribuição do cirurgião-dentista (CD) no incremento do diagnóstico precoce da hanseníase, este estudo tem como objetivo conhecer as principais características epidemiológicas dos portadores de hanseníase com ênfase à área de localização das lesões no município de Cáceres, MT, no período de 2001 a 2006.

\section{Métodos}

Trata-se de um estudo transversal do registro de indivíduos com hanseníase, notificados no município de Cáceres-MT, no período de 2001 a 2006.

Os dados foram coletados dos prontuários de todos os pacientes diagnosticados com hanseníase em Cáceres. As anotações contidas nas fichas de assistência médica e nas fichas de prevenção de incapacidades foram agrupadas em um formulário específico.

A área de localização das lesões foi definida como variável dependente e categorizada, em lesão em área visível que inclui: face, pavilhão auricular ou membros superiores (MMSS) e lesão em área nãovisível para demais regiões do corpo. As variáveis independentes foram classificadas como: relacionadas ao indivíduo, ao serviço de saúde e à doença. Para comparação de médias e ajustamentos, a idade foi mantida como variável contínua. Para análise estratificada, a idade foi categorizada em menores de 15 anos e igual ou maiores de 15 anos.
Foi elaborado e validado com dupla digitação um banco de dados no programa EpiInfo 3.3.2. Realizou-se análise de variância (ANOVA) para comparação das médias de idade segundo sexo e variável dependente, lesão em área visível e lesão em área nãovisível. Para verificar associação entre as variáveis preditoras e a variável dependente utilizou-se regressão múltipla logística com nível de significância de 95\%.

Para a identificação de possíveis efeitos de confusão ou interação em relação à associação entre a variável dependente e demais variáveis, realizou-se análise estratificada, segundo Mantel-Haenszel, das variáveis que poderiam apresentar tais efeitos.

Esta pesquisa foi aprovada pelo Comitê de Ética e Pesquisa do Hospital Universitário Júlio Müller (protocolo Nº 289/CEPHUJM/06).

O município de Cáceres está localizado na região sudoeste do Estado de Mato Grosso, a 210 km da capital Cuiabá. Com uma população estimada para 2007 de 91.714 habitantes ${ }^{15}$, este município foi classificado como uma região de alta prevalência, segundo os parâmetros da Organização Mundial de Saúde, por apresentar 7,52 doentes em tratamento por 10.000 habitantes em $2005^{1}$.

Embora a descentralização das ações de controle da hanseníase no município de Cáceres tenha sido iniciada em 2001, o diagnóstico permanece centralizado no ambulatório de referência em hanseníase, onde também são realizados os exames laboratoriais. O município apresenta 10 unidades de saúde da família que realizam a notificação, o acompanhamento e a administração da poliquimioterapia (PQT) para os pacientes com residência distante do ambulatório. O município também está estruturado com 8 unidades de saúde com atendimento odontológico, dos quais 4 fazem parte do Programa de Saúde da Família. Os serviços odontológicos na rede privada são realizados por 58 profissionais: destes, 21 atendem no serviço público e somente 3 são exclusivos da rede pública. 


\section{Resultados}

No período de $1^{\circ}$ de janeiro de 2001 a 31 de dezembro de 2006 foram diagnosticados 609 pessoas com hanseníase no município de Cáceres-MT, dos quais 206 (33,9\%) apresentaram lesão em área visível no momento do diagnóstico. Na Figura 1 observa-se que a proporção de lesões em área visível manteve-se abaixo da proporção de lesões em área não-visível durante o período estudado, porém após o ano de 2004 ocorreu inversão de tendência. Enquanto a proporção de lesões em área visível aumentou $9,7 \%$ até o ano de 2006, a proporção de lesões em área não-visível diminuiu 9,7\% até o ano de 2006. Na série estudada, o coeficiente de detecção mostrou tendência ao declínio, com redução de 17,5 casos/ 10.000 habitantes em 2001, para 7,2 casos/ 10.000 habitantes em 2006.

Dentre os 206 pacientes com lesão em área visível, 135 indivíduos $(65,5 \%)$ correspondiam ao sexo masculino e 71 indivíduos $(34,5 \%)$ ao sexo feminino. Os pacientes do sexo masculino apresentaram idade média próxima de 41 anos (5 mínima, 89 máximo), enquanto a idade média das mulheres foi 37 anos (7 mínima, 76 máximo). Ainda que 356 (58,5\%) indivíduos do estudo sejam do sexo masculino, não foi verificada diferença estatisticamente significante entre a média de idade segundo sexo e área da lesão (Figura 2).

Observa-se na Figura 3 que a região do corpo com maior número de lesões visíveis foi o braço (134), seguida da face (56) e das mãos (15), enquanto somente 1 indivíduo apresentou lesões no pavilhão auricular.

$\mathrm{Na}$ análise bivariada (odds ratio bruto), observa-se que indivíduos do sexo masculino apresentaram probabilidade $69 \%$ maior de manifestar lesão em área visível se comparado ao sexo feminino (Tabela 1). Para o grupo de indivíduos com idade menor que 15 anos, a probabilidade de apresentar lesões em área visível foi de $63 \%$, entretanto, sem significância estatística $(\mathrm{p}=0,189)$. Quanto às variáveis relacionadas ao serviço, a probabilidade de os pacientes apresentarem lesões em área visível, no momento do diagnóstico, terem sido diagnosticados

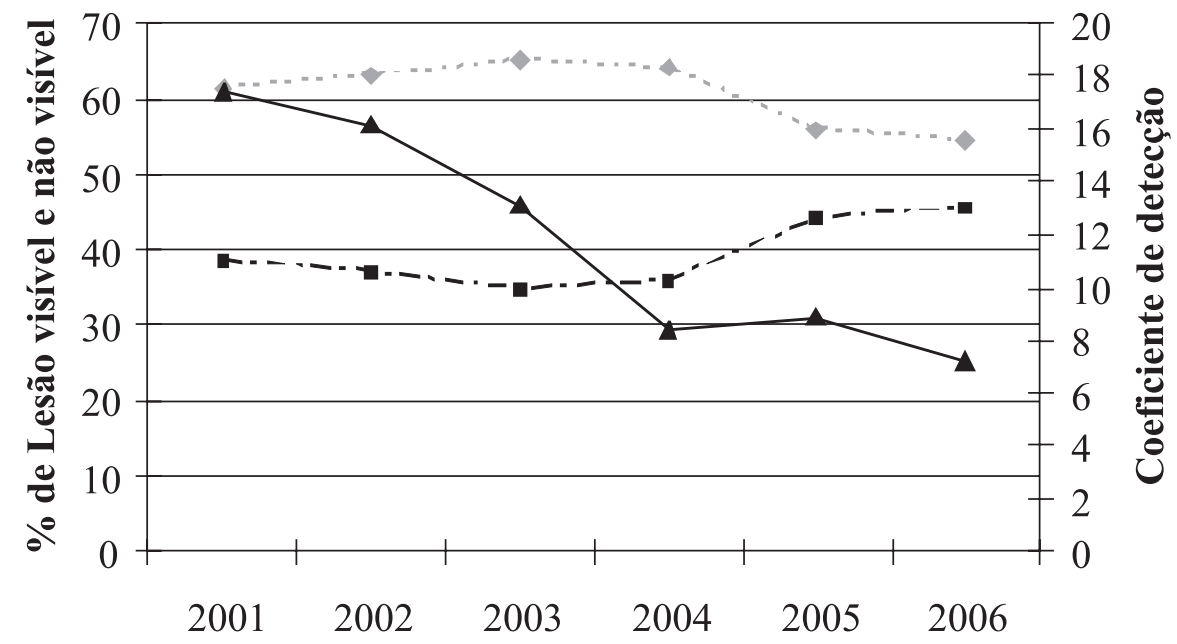

Ano

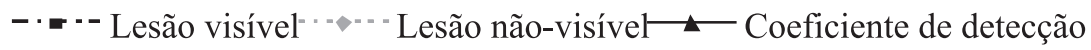

Figura 1 - Distribuição percentual de lesões em área visível e não-visível e coeficiente de detecção de hanseníase no município de Cáceres-MT (2001 a 2006).

Figure 1 - Distribution of lesions in visible and non-visible areas and detection rate of leprosy in the city of Cáceres, state of Mato Grosso, Brazil (2001 to 2006). 


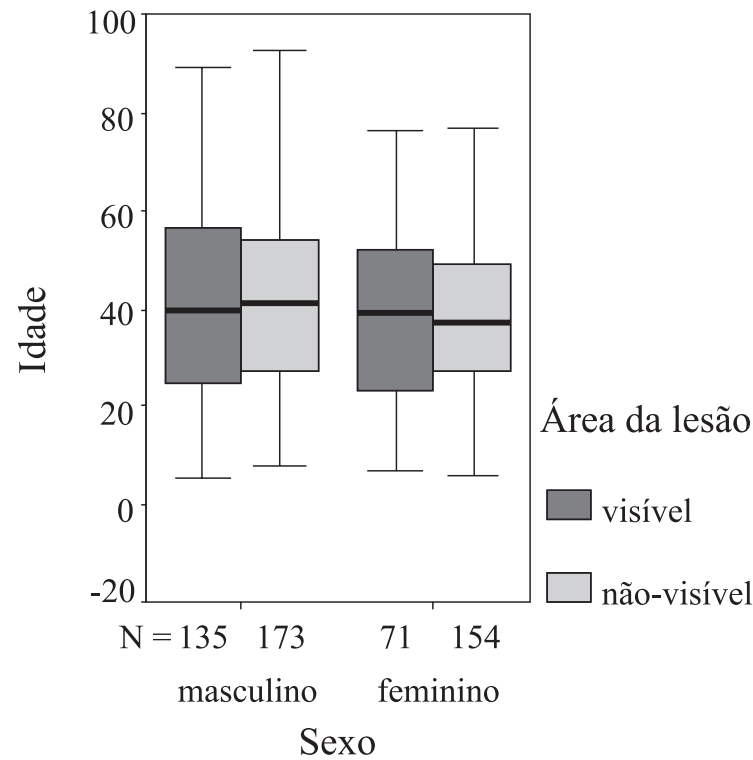

Figura 2 - Distribuição dos casos de hanseníase por idade e área da lesão segundo o sexo no município de Cáceres, estado de Mato Grosso, Brasil (2001 a 2006).

Figure 2 -Distribution of leprosy cases by age and site of lesion according to gender in the city of Caceres, state of Mato Grosso, Brazil (2001 to 2006).

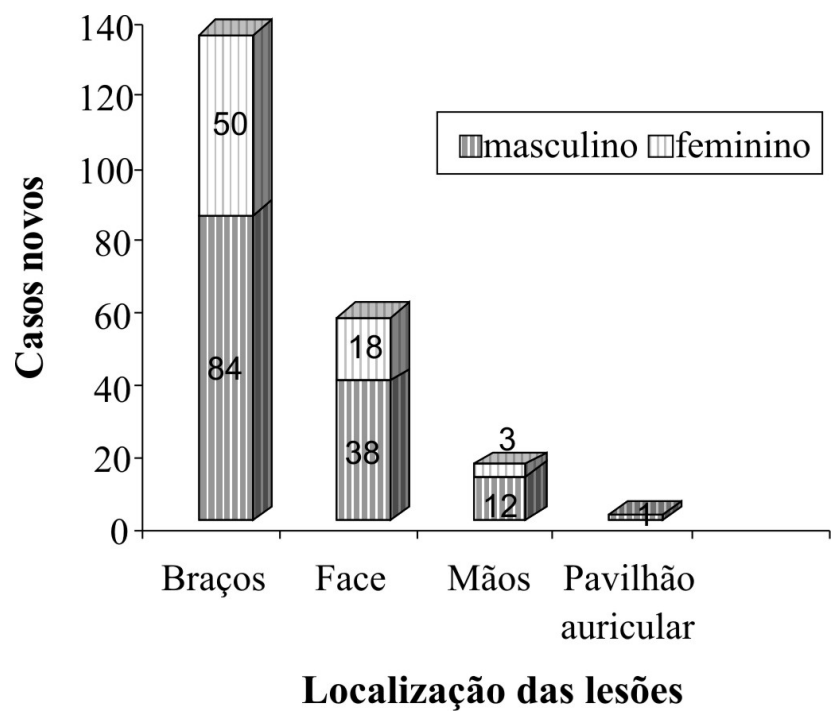

Figura 3 - Distribuição dos casos de hanseníase com lesões em áreas visíveis segundo local de manifestações das lesões e sexo. Cáceres-MT (2001 a 2006).

Figure 3 - Distribution of leprosy cases with lesions in visible areas by site of manifestation and sex. Caceres, state of Mato Grosso, Brazil (2001 to 2006).

e tratados na unidade de referência em dermatologia foi $49 \%$ maior se comparados aos tratados nas unidades de Saúde da Família ( $\mathrm{p}=0,043)$. A probabilidade de os indivíduos diagnosticados apresentarem lesões em área visível decorridos seis meses do início dos sinais e sintomas foi de $25 \%$, sem significância estatística $(\mathrm{p}=0,316)$. Em relação às variáveis escolaridade, zona de residência, modo de detecção e modo de en- 
trada, também não se observou associação significativa com a variável em estudo.

Verifica-se ainda que, entre os indivíduos detectados com baciloscopia positiva, a probabilidade de apresentarem lesões em área visível foi 3 vezes aquela observada entre os indivíduos com baciloscopia negativa. Do mesmo modo, entre os indivíduos multibacilares a probabilidade de diagnóstico de casos com lesão em área visível foi 3 vezes aquela observada entre os indivíduos paucibacilares (OR = 3,32: IC 95\%: 2,20 - 5,03). Entre os indivíduos diagnosticados com a forma clínica virchowiana, tal probabi- lidade foi 5 vezes aquela observada entre os indivíduos diagnosticados com a forma clínica indeterminada ( $\mathrm{OR}=5,09$; IC 95\%: 2,72 - 9,59), enquanto entre os indivíduos com a forma clínica dimorfa a probabilidade de apresentarem lesões em área visível foi 3 vezes aquela observada entre os indivíduos com forma clínica indeterminada $(\mathrm{OR}=3,22$; IC 95\%: 1,71-6,09). Considerando-se o tipo de lesão e tendo por referência máculas, a probabilidade de a lesão ser uma pápula ou nódulo e estar em área visível foi cinco vezes aquela observada entre as lesões maculares $(\mathrm{OR}=5,78 ;$ IC 95\%: 2,13 - 16,53) e quase o

Tabela 1 - Análise bivariada de casos de hanseníase com lesão em área visível e não-visível segundo variáveis relacionadas ao indivíduo, ao serviço e à doença. Cáceres-MT (2001 a 2006).

Table 1 - Bivariate analysis of leprosy cases with lesions in visible and non-visible areas by variables related to the individual, healthcare facility and disease. Caceres, state of Mato Grosso, Brazil (2001 to 2006).

\begin{tabular}{|c|c|c|c|c|c|c|}
\hline \multirow[b]{2}{*}{ Variáveis } & \multicolumn{2}{|c|}{ Lesão em área visível } & \multicolumn{2}{|c|}{ Lesão em área não visível } & \multirow{2}{*}{$\begin{array}{l}\text { OR Bruto } \\
\text { (IC 95\%) }\end{array}$} & \multirow[b]{2}{*}{ p-valor } \\
\hline & $\mathrm{N}$ & $\%$ & $\mathrm{~N}$ & $\%$ & & \\
\hline \multicolumn{7}{|l|}{ Relacionadas ao indivíduo } \\
\hline \multicolumn{7}{|l|}{ Sexo } \\
\hline Masculino & 135 & 43,8 & 173 & 56,2 & $1,69(1,16-2,47)$ & 0,004 \\
\hline Feminino & 71 & 31,6 & 154 & 68,4 & 1,0 & \\
\hline \multicolumn{7}{|l|}{ Idade em anos } \\
\hline$<15$ anos & 15 & 50,0 & 15 & 50,0 & $1,63(0,74-3,62)$ & 0,189 \\
\hline$\geqq 15$ anos & 191 & 38,0 & 312 & 62,0 & 1,0 & \\
\hline \multicolumn{7}{|l|}{ Escolaridade } \\
\hline $\begin{array}{l}\text { Até ensino fundamental } \\
\text { completo }\end{array}$ & 135 & 39,8 & 204 & 60,2 & $1,03(0,68-1,57)$ & 0,871 \\
\hline Ensino médio e superior & 57 & 39,0 & 89 & 61,0 & 1,0 & \\
\hline \multicolumn{7}{|l|}{ Zona de residência } \\
\hline Urbana & 151 & 38,6 & 240 & 61,4 & $0,98(0,65-1,49)$ & 0,935 \\
\hline Rural & 55 & 39,0 & 86 & 61,0 & 1,0 & \\
\hline \multicolumn{7}{|l|}{ Relacionadas ao serviço } \\
\hline \multicolumn{7}{|l|}{ Tipo de unidade de saúde } \\
\hline Unidade de Referência & 152 & 41,5 & 214 & 58,5 & $1,49(0,99-2,23)$ & 0,043 \\
\hline PSF & 54 & 32,3 & 113 & 67,7 & 1,0 & \\
\hline \multicolumn{7}{|l|}{ Modo de detecção } \\
\hline Encaminhamento & 54 & 42,2 & 74 & 57,8 & $1,57(0,88-2,82)$ & 0,104 \\
\hline Detecção espontânea & 111 & 39,1 & 173 & 60,9 & $1,38(0,83-2,31)$ & 0,187 \\
\hline Outros & 32 & 31,7 & 69 & 68,3 & 1,0 & \\
\hline \multicolumn{7}{|l|}{ Modo de entrada } \\
\hline Outros Reingressos & 10 & 45,5 & 12 & 54,5 & $1,33(0,52-3,73)$ & 0,513 \\
\hline Caso Novo & 196 & 38,5 & 313 & 61,5 & 1,0 & \\
\hline \multicolumn{7}{|l|}{ Tempo de detecção } \\
\hline$>6$ meses & 76 & 42,7 & 102 & 57,3 & $1,25(0,79-1,96)$ & 0,316 \\
\hline$\leq 6$ meses & 64 & 37,4 & 107 & 62,6 & 1,0 & \\
\hline
\end{tabular}


Tabela 1 (cont.) - Análise bivariada de casos de hanseníase com lesão em área visível e não-visível segundo variáveis relacionadas ao indivíduo, ao serviço e à doença. Cáceres-MT (2001 a 2006).

Table 1 (cont'd) - Bivariate analysis of leprosy cases with lesions in visible and non-visible areas by variables related to the individual, healthcare facility and disease. Caceres, state of Mato Grosso, Brazil (2001 to 2006).

\begin{tabular}{|c|c|c|c|c|c|c|}
\hline \multirow[b]{2}{*}{ Variáveis } & \multicolumn{2}{|c|}{ Lesão em área visível } & \multicolumn{2}{|c|}{ Lesão em área não visível } & \multirow{2}{*}{$\begin{array}{l}\text { OR Bruto } \\
\text { (IC 95\%) }\end{array}$} & \multirow[b]{2}{*}{ p-valor } \\
\hline & $\mathrm{N}$ & $\%$ & $\mathrm{~N}$ & $\%$ & & \\
\hline \multicolumn{7}{|c|}{ Relacionadas à doença } \\
\hline \multicolumn{7}{|c|}{ Classificação Operacional } \\
\hline Paucibacilar & 119 & 30,7 & 268 & 69,3 & 1,0 & \\
\hline Multibacilar & 87 & 59,6 & 59 & 40,4 & $3,32(2,20-5,03)$ & $<0,001$ \\
\hline \multicolumn{7}{|l|}{ Baciloscopia } \\
\hline Negativo & 122 & 31,3 & 268 & 68,7 & 1,0 & \\
\hline Positivo & 81 & 58,3 & 58 & 41,7 & $3,07(2,02-4,67)$ & $<0,001$ \\
\hline \multicolumn{7}{|l|}{ Forma Clínica } \\
\hline Indeterminada & 39 & 26,4 & 109 & 73,6 & 1,0 & \\
\hline Tuberculóide & 78 & 33,2 & 157 & 66,8 & $1,39(0,86-2,25)$ & 0,158 \\
\hline Dimorfa & 38 & 53,5 & 33 & 46,5 & $3,22(1,71-6,09)$ & $<0,001$ \\
\hline Virchowiana & 51 & 64,6 & 28 & 35,4 & $5,09(2,72-9,59)$ & $<0,001$ \\
\hline \multicolumn{7}{|l|}{ Tipo de lesão de pele } \\
\hline Mácula & 136 & 36,6 & 236 & 63,4 & 1,0 & \\
\hline Placa & 27 & 52,9 & 24 & 47,1 & $1,95(1,04-3,66)$ & 0,024 \\
\hline Pápula ou nódulo & 20 & 76,9 & 6 & 23,1 & $5,78(2,13-16,53)$ & $<0,001$ \\
\hline \multicolumn{7}{|l|}{ Número de lesão } \\
\hline Única & 80 & 26,8 & 219 & 73,2 & 1,0 & \\
\hline Múltipla & 114 & 56,4 & 88 & 43,6 & $3,55(2,39-5,27)$ & $<0,001$ \\
\hline \multicolumn{7}{|l|}{ Pigmentação da lesão } \\
\hline Hipopigmentada & 72 & 34,6 & 136 & 65,4 & 1,0 & \\
\hline Hiperpigmentada & 78 & 45,3 & 94 & 54,7 & $1,57(1,01-2,42)$ & 0,033 \\
\hline \multicolumn{7}{|l|}{ Incapacidade Física } \\
\hline Sim & 76 & 55,1 & 62 & 44,9 & $2,66(1,73-4,08)$ & $<0,001$ \\
\hline Não & 102 & 31,6 & 221 & 68,4 & 1,0 & \\
\hline \multicolumn{7}{|c|}{ Local de incapacidade física } \\
\hline Ocular e MMSSa & 46 & 71,9 & 18 & 28,1 & $3,47(1,57-7,73)$ & $<0,001$ \\
\hline Outros & 28 & 42,4 & 38 & 57,6 & 1,0 & \\
\hline
\end{tabular}

Nota: ${ }^{(a)}$ upper limbs/ membros superiores

dobro entre as lesões do tipo placa $(\mathrm{OR}=1,95$; IC 95\%: 1,04 - 3,66). A probabilidade de as lesões serem múltiplas e com localização em área visível foi três vezes aquela observada entre as lesões únicas (OR = 3,55; IC 95\%: 2,39 - 5,27) e estatisticamente significante em relação a se manifestarem hiperpigmentadas $(\mathrm{OR}=1,57$; IC 95\%: 1,01-2,42).

Entre os indivíduos diagnosticados com incapacidade física, a probabilidade de apresentarem lesões em área visível foi duas vezes aquela observada nos indivíduos sem incapacidade ( $\mathrm{OR}=2,66$; IC 95\%: 1,73 4,08 ) e tal probabilidade entre aqueles que apresentaram a área ocular ou dos MMSS comprometida foi três vezes aquela observada entre indivíduos que manifestaram incapacidade em outros locais $(\mathrm{OR}=3,47$; IC 95\%: 1,57 - 7,73).

Em razão da identificação da variável classificação operacional ser um fator de confundimento, a análise multivariada foi estratificada para as categorias paucibacilar e multibacilar, como está descrito na Tabela 2.

Observa-se, após a análise estratificada e ajustamento por idade, que a associação verificada entre sexo e presença de lesão em 
Tabela 2 - Modelos de regressão múltipla logística de casos de hanseníase estratificados por classificação operacional segundo área de localização das lesões, e variáveis relacionadas ao indivíduo (sexo e idade), ao serviço (tempo de detecção e tipo de unidade de saúde) e à doença (incapacidade física e local de incapacidade física). Cáceres - MT (2001 a 2006).

Table 2 - Models of multiple logistic regression of leprosy cases stratified by operational classification and site of lesion, and variables related to the individual (gender and age), healthcare facility (time of detection and type of healthcare facility) and disease (physical disability and site of physical disability). Caceres - MT (2001 to 2006).

\begin{tabular}{|c|c|c|c|c|}
\hline \multirow[b]{2}{*}{ Variáveis } & \multicolumn{2}{|c|}{ Paucibacilar } & \multicolumn{2}{|c|}{ Multibacilar } \\
\hline & $\begin{array}{l}\text { OR Ajustado } \\
\text { (IC 95\%) }\end{array}$ & $\mathrm{p}$-valor & $\begin{array}{l}\text { OR Ajustado } \\
\text { (IC 95\%) }\end{array}$ & $\mathrm{p}$-valor \\
\hline \multicolumn{5}{|c|}{ Relacionadas ao indivíduo } \\
\hline \multicolumn{5}{|l|}{ Sexo } \\
\hline Masculino & $1,43(0,92-2,20)$ & 0,111 & $1,26(0,59-2,71)$ & 0,549 \\
\hline Feminino & 1,0 & & 1,0 & \\
\hline \multicolumn{5}{|l|}{ Idade em anos ${ }^{b}$} \\
\hline$<15$ anos & $2,29(0,97-5,40)$ & 0,058 & $2,46(0,15-40,12)$ & 0,527 \\
\hline$\geq 15$ anos & 1,0 & & 1,0 & \\
\hline \multicolumn{5}{|c|}{ Relacionadas ao serviço } \\
\hline \multicolumn{5}{|c|}{ Tempo de detecçãoc } \\
\hline$>6$ meses & $1,58(0,84-2,94)$ & 0,153 & $2,56(0,66-10,00)$ & 0,176 \\
\hline$\leq 6$ meses & 1,0 & & 1,0 & \\
\hline \multicolumn{5}{|c|}{ Tipo de unidade de saúde ${ }^{d}$} \\
\hline Referência & $1,66(0,37-7,49)$ & 0,511 & $\begin{array}{c}12,68(1,13- \\
142,86)\end{array}$ & 0,040 \\
\hline PSF & 1,0 & & 1,0 & \\
\hline \multicolumn{5}{|c|}{ Relacionadas à doença } \\
\hline \multicolumn{5}{|c|}{ Incapacidade Físicae } \\
\hline Sim & $2,29(1,26-4,16)$ & 0,006 & $2,55(1,07-6,04)$ & 0,034 \\
\hline Nao & 1,0 & & 1,0 & \\
\hline \multicolumn{5}{|c|}{ Local de incapacidade física $^{f}$} \\
\hline Ocular e MMSSg & $3,03(1,06-8,64)$ & 0,039 & $5,10(1,41-18,39)$ & 0,013 \\
\hline Outros & 1,0 & & 1,0 & \\
\hline
\end{tabular}

Notas: ${ }^{(a)}$ adjusted by age / ajustado por idade

(b) adjusted by gender, age, area of residence, schooling, and type of healthcare facility / ajustado por sexo, idade, residência, escolaridade, unidade de saúde

(c) adjusted by gender, age, and presence of physical disability / ajustado por sexo, idade, presença de incapacidade física

(d) adjusted by gender, age, area of residence, schooling, and site of physical disability / ajustado por sexo, idade, residência, educação, local de incapacidade física

(e) adjusted by gender, age, area of residence, schooling, and type of healthcare facility /ajustado por sexo, idade, residência, educação, unidade de saúde.

(f) adjusted by gender, age, and area of residence / ajustado por sexo, idade, residência

(g) upper limbs/ membros superiores

área visível perdeu significância, tanto para os casos paucibacilares $(\mathrm{p}=0,111)$, como multibacilares $(\mathrm{p}=0,549)$. Quanto à variável idade, após ajustamento por sexo, local de residência, escolaridade e tipo de unidade de saúde, a associação não se mostrou significativa $(\mathrm{p}=0,527)$.
Entre os pacientes multibacilares diagnosticados após 6 meses do início dos sinais e sintomas, a probabilidade de apresentarem lesão em área visível foi duas vezes aquela observada entre aqueles com menor tempo de diagnóstico. Contudo, essa associação não foi estatisticamente significante, 
mesmo após ajustamento por sexo, idade e presença de incapacidade física $\left(\mathrm{OR}_{\text {ajust }}=\right.$ 2,56; IC 95 \%: 0,66 - 10,00).

Tal probabilidade de apresentar lesão em área visível entre os pacientes multibacilares com atendimento na unidade de referência foi doze vezes a observada entre aqueles com atendimento na unidade de saúde da família, independente de sexo, idade, residência, escolaridade e local de incapacidade física $\left(\mathrm{OR}_{\text {ajust }}=12,68\right.$; IC $95 \%$ : 1,13 - 142,86).

Pode-se observar que a probabilidade, entre os indivíduos paucibacilares e multibacilares, diagnosticados com incapacidade física, de apresentarem lesão em área visível foi duas vezes aquela observada entre aqueles que não apresentavam incapacidade física no momento do diagnóstico, mesmo após ajustamento por sexo, idade, residência, escolaridade e unidade de saúde. Quando a incapacidade física estava localizada na região ocular e nos MMSS, tal probabilidade foi três vezes aquela observada entre os pacientes paucibacilares $\left(\mathrm{OR}_{\text {ajust }}=3,03\right.$; IC $95 \%$ : $1,06-8,64)$ e cinco vezes a observada entre os pacientes multibacilares com incapacidade física em outra área do corpo $\left(\mathrm{OR}_{\text {ajust }}=\right.$ 5,10; IC 95\%: 1,41 - 18,39), após ajustamento por sexo, idade e local de residência.

\section{Discussão e Conclusão}

A capacidade de identificar lesões dermatológicas como suspeitas de hanseníase e o encaminhamento para um serviço de saúde, onde o diagnóstico possa ser realizado corretamente, é uma importante habilidade que se espera dos profissionais de saúde, principalmente dos municípios endêmicos. Este estudo mostra a importância e a possibilidade de envolvimento dos CDs nas ações de eliminação da hanseníase.

Tendo como pressuposto a integralidade no atendimento do paciente, no sentido de "a integralidade como um traço da boa medicina" ${ }^{16}$, o CD da rede de saúde pública e/ou privada, durante uma consulta, não deve reduzir o indivíduo a um aparelho ou sistema biológico ${ }^{17}$, mas sim, ter um olhar que vá além do reducionismo bucal, buscando reconhecer as necessidades de saúde não verbalizadas ou explícitas. Assim, o reconhecimento e a identificação de lesões na pele localizadas em áreas expostas representam uma ação de responsabilidade no cuidado à saúde.

O diagnóstico da hanseníase foi simplificado nos últimos anos como estratégia de acessibilidade, não sendo essencial a realização de exames laboratoriais. $\mathrm{O}$ enfoque está no reconhecimento dos sinais cardinais e exame clínico criterioso das mãos, pés, olhos, nervos periféricos e testes de sensibilidade ${ }^{18}$.

Segundo os resultados deste estudo, as lesões de pele de hanseníase podem se manifestar em braços, face e mãos, podendo estar presentes tanto em indivíduos do sexo masculino quanto do feminino, adultos ou crianças, ou seja, independem de idade e sexo.

Em estudo realizado na Índia, crianças menores de 15 anos apresentaram maior proporção de lesões nos membros inferiores, superiores, face e tórax, respectivamente; entretanto, a somatória das lesões com localização em membros superiores e face mostraram-se superiores às demais áreas, não evidenciando diferença significante em relação ao sexo ${ }^{10}$. Em indivíduos acima de 15 anos não foram observadas diferenças entre o tipo de hanseníase e o local das lesões (Kumar et al. ${ }^{19}$ ).

Ponnighaus et al. ${ }^{7}$, analisando a distribuição de lesões únicas em uma população da África, observaram maior proporção de lesões em face e atrás dos braços, independentemente da idade, porém observou diferença de distribuição segundo o sexo. As lesões em áreas da face e braço foram mais comuns em indivíduos adultos do sexo feminino, e aquelas atrás do braço, mais comuns aos indivíduos do sexo masculino. No Brasil, Avelleira et al. ${ }^{8}$ observaram que a localização de lesões únicas, em casos paucibacilares, predominou no sexo feminino principalmente em MMSS e face, com expressivo percentual de lesões em face nas crianças. 
O padrão de distribuição e localização das lesões pode refletir alguns fatores comportamentais, culturais ou ambientais e se considera a hipótese de que a área de localização de uma lesão primária possa ser a manifestação de uma outra porta de entrada do M. leprae no organismo ${ }^{10}$, pois se discute a transmissão indireta, onde reservatórios do bacilo, fora do corpo humano, possam existir e agir em diferentes níveis de transmissão ou evolução da doença ${ }^{20}$.

Katoch et al. ${ }^{9}$ relatam ter encontrado 94\% das lesões em áreas expostas, incluindo aquelas com localização nas pernas e pés, sendo a maioria máculas e hiperpigmentadas. Lesões do tipo mácula hipopigmentadas e em pequeno número são comuns em crianças com idade inferior a 15 anos, em regiões expostas do corpo ${ }^{21}$, nas formas clínicas indeterminada, tuberculóide ${ }^{22}$ ou dimorfa ${ }^{23}$, devendo ser consideradas como suspeitas de hanseníase.

Quando um indivíduo, apresentando lesão de pele em área visível, procura atendimento odontológico, o tipo da lesão, o número e a presença ou não de pigmentação, são características que podem se somar para uma suspeita diagnóstica, principalmente se hiperpigmentadas e múltiplas, comuns às formas clínicas dimorfa e virchowiana.

Sendo a lesão de hanseníase com localização na pele um sinal cardinal para a suspeita diagnóstica, espera-se que, uma vez em áreas expostas do corpo, também possam ser mais facilmente notadas por profissionais de saúde, como os CD. Reconhecer uma lesão por suas características clínicas, ou visualizá-la, mesmo quando em locais expostos, depende de um olhar sensível e atento, da disposição em se realizar aquilo que parece não ser a demanda usual no desenvolvimento do seu trabalho, mas quando está ao seu alcance, essa atitude demonstra preocupação com a saúde e a qualidade de vida do paciente.

Para o controle da hanseníase podese observar uma preocupação dos órgãos governamentais e não-governamentais em capacitação dos profissionais de saúde para o alcance da meta de eliminação proposta pela $\mathrm{OMS}^{24}$. Todavia, o conhecimento dos CD sobre hanseníase é outro aspecto relevante a se considerar.

Sabe-se que a demora no diagnóstico após o início dos sinais e sintomas pode influenciar no desenvolvimento de incapacidades ${ }^{25}$, ainda que haja evidências de que idade, forma clínica e sexo possam agir como fatores de risco independentes para a presença de incapacidade ${ }^{26}$. $\mathrm{O}$ atraso no diagnóstico pode apresentar causas diferentes em lugares distintos ${ }^{27}$.

No período estudado, 204 casos $(33,5 \%)$ foram diagnosticados 6 meses após o aparecimento dos sintomas; no entanto, o número de casos sem esta informação foi maior: 211 (34,6\%), apontando a pouca importância dada pelo serviço de vigilância ao tempo de início dos sintomas até o diagnóstico. No estudo, a variável tempo do início dos sintomas até o diagnóstico não mostrou associação significante com lesão em área visível, mesmo após ajustamento. Todavia, a coleta deste dado nos prontuários pode ser uma limitação para a análise, em razão da dependência da valorização desta informação pelo profissional de saúde que realizou a anamnese.

Além das lesões na pele, os casos de hanseníase com diagnóstico tardio podem se manifestar com alterações faciais, como paralisia, madarose e infiltrações na testa, nariz e orelhas, comprometendo muitas vezes as expressões faciais. Opromolla et al. ${ }^{28}$, ao discutirem fatores relacionados ao diagnóstico tardio da hanseníase, fazem referência ao desconhecimento de muitos profissionais de saúde sobre as manifestações da hanseníase e acrescentam também a pouca importância que os profissionais de saúde dão as alterações faciais que ocorrem na velhice, passando desapercebidas, quer estando acentuadas ou não, e que podem expressar algum sinal patológico. Ainda que tais sinais se manifestem em casos avançados, estes refletem a manutenção da endemia.

Nesse estudo, as lesões incapacitantes em áreas visíveis ao $\mathrm{CD}$, como mãos, braços e face, inclusive olhos, não foram somadas 
às lesões de pele. No entanto, quando os indivíduos com hanseníase apresentam incapacidades físicas nestas áreas do corpo aumenta a possibilidade de o CD suspeitar da presença de hanseníase.

Em municípios como Cáceres, onde a temperatura média anual no verão é de $32^{\circ} \mathrm{C}$, e ainda considerando as características culturais pela proximidade do rio Paraguai, no que diz respeito ao vestuário, é muito comum o uso de camisetas e shorts, independente do ambiente freqüentado. Esse fato contribui para a possibilidade de o CD identificar lesões em áreas visíveis durante uma consulta de rotina, também favorecida pelos retornos decorrentes do tratamento e acompanhamento odontológico.

Quando hábitos culturais ou problemas socioeconômicos interferem na oportunidade de detecção da hanseníase, como em mulheres da Turquia ${ }^{29}$ e Índia, onde mulheres são recrutadas para examinar mulheres ${ }^{30}$, o tempo de detecção pode ser duas vezes mais demorado ${ }^{31} \mathrm{e}$ a forma clinica virchowiana pode ser observada com maior freqüência. Al-Kadari et al..$^{32}$, em estudo realizado no Kuwait entre 3 etnias (Ásia-Índia, Oriental e Árabe) sugere a influência dos fatores culturais pelo maior tempo de atraso no diagnóstico entre as mulheres árabes. Como conseqüência, observa-se maior proporção de incapacidades ${ }^{29}$, maior estigma e isolamento ${ }^{33} \mathrm{e}$, por conseguinte, a não aderência ao tratamento ${ }^{19}$.

Embora o bacilo seja transmitido através das vias aéreas superiores, o risco de um CD ser infectado durante o tratamento de um paciente ainda não tratado é pouco discutido. Alfieri et al. ${ }^{34}$, estudando a manifestação de lesões hansênicas no palato de indivíduos com hanseníase tuberculóide reacional e dimorfa, não considerou a mucosa bucal como importante fonte de eliminação do bacilo. Ainda que as lesões na mucosa apresentem grande quantidade do bacilo, não devem ser contagiantes como casos com a forma clínica virchowiana. Opromolla et al. ${ }^{35}$ atentam para o fato de que, nos casos reacionais, os surtos têm a tendência a regredir espontaneamente, assim como a baciloscopia de pele ou mucosa tende a negativar.

Considerando-se também o fato de os CDs usarem equipamentos de proteção individual, como luvas e máscaras, durante procedimentos de rotina, e de os indivíduos em tratamento PQT deixarem de ser casos contagiantes $^{6}$, o risco de transmissão do bacilo ao $\mathrm{CD}$, durante a realização do tratamento odontológico, provavelmente é pequeno. Acrescenta-se a isso outro fator importante para que ocorra a manifestação da doença: a susceptibilidade do indivíduo ${ }^{6}$.

De Abreu et al. ${ }^{36}$, estudando a manifestação de lesões hansênicas na cavidade bucal de pacientes multibacilares não tratados, argumentam a necessidade de médicos e CDs se protegerem quando realizarem procedimentos invasivos nessa área. Assim, ainda que os CDs possam estar mais expostos em áreas endêmicas, a relevância na identificação de casos suspeitos de hanseníase pelo $\mathrm{CD}$, durante o atendimento odontológico, parece trazer indubitavelmente maior benefício para os pacientes do que contribuir para a redução de risco de adoecimento nesses profissionais.

No processo de descentralização e desconcentração das ações programáticas de hanseníase é necessário aos municípios endêmicos, não só a autonomia, como foi viabilizada pela NOB/96, mas a decisão de gestão, afim de que todos os profissionais da rede básica de saúde contribuam para o diagnóstico da doença e do tratamento. Assim, entre outras medidas que visam implementar as ações de controle, torna-se necessário, o fortalecimento de programas de capacitação dos profissionais de saúde em geral ${ }^{37}$.

No Brasil, o alcance da meta de eliminação da hanseníase como problema de saúde pública tornou-se um desafio para os municípios. Espera-se que todos os municípios endêmicos até 2010 tenham menos de um caso de hanseníase em tratamento a cada 10.000 habitantes ${ }^{24}$.

No município de Cáceres, o estudo de tendência para o período de 2001 a 2006, 
evidenciou um declínio no coeficiente de detecção de casos novos de 17,5 casos por 10.000 habitantes, em 2001, para 7,2 casos por 10.000 habitantes em 2006. Embora tenha ocorrido expressiva redução, por esse indicador o município pode ser considerado como um dos mais endêmicos do Estado de Mato Grosso. Para o padrão estabelecido pela OMS, Cáceres possui quase o dobro de casos detectados para ser considerado como hiperendêmica $(\geq 4,0$ casos / 10.000 habitantes). O coeficiente de detecção de casos novos para a população com faixa etária menor de 15 anos foi de 1,03 casos/ 10.000 habitantes, índice superior ao valor de 0,9 casos por 10.000 habitantes observados para a Região Centro-Oeste em dezembro de 2006, assim como ao parâmetro que caracteriza esta cidade como hiperendêmico $(\geq 1 \text { caso } / 10.000 \text { habitantes })^{38}$. Segundo o Plano Nacional de Eliminação da Hanseníase em nível municipal 2006-2010, esses valores revelam a necessidade de focalizar e agilizar o diagnóstico de hanseníase. Casos novos dessa doença em menores de 15 anos podem indicar casos não-diagnosticados e não-assistidos pelas unidades de saúde ${ }^{39}$.

$\mathrm{Na}$ análise da proporção de lesões em área visível e não-visível no período de estudo, observou-se uma inversão de tendência a partir de 2004. Deste ano a 2006, o percentual de lesões em área visível, em relação ao valor total, elevou-se para $27 \%$, enquanto o percentual de lesões não-visíveis reduziu $16 \%$. Ainda que tenha ocorrido redução na detecção da hanseníase em Cáceres, esta inversão na tendência, segundo área de localização de lesões, sugere que aqueles que apresentam as lesões mais facilmente visíveis (membros superiores, face e pavilhão auricular), independente do tipo de serviço de saúde, estão sendo detectados em maior proporção. Dessa forma, em detrimento da característica endêmica do município, a queda no coeficiente de detecção pode ser decorrente de deficiência na capacidade de diagnóstico ${ }^{40}$. Possivelmente, o aumento de informações relativas às características da doença, pela mídia e pelas campanhas educativas, tem favorecido a identificação de lesões visíveis pelos profissionais de saúde e pela população em geral, em detrimento daquelas não-visíveis e que necessitam de um exame clínico mais acurado para sua detecção.

Em Cáceres, embora 73,3 \% das unidades básicas realizem a distribuição da medicação para o tratamento de hanseníase, o diagnóstico e o atendimento dos casos mais graves continuam centralizados no ambulatório de referência, o que sugere fragilidades na descentralização das atividades de controle da doença. Segundo Oliveira et al. ${ }^{41} \mathrm{em}$ Cáceres tem havido pequeno incremento do número de contatos examinado para cada caso diagnosticado; no entanto, há uma perda de em média 3 casos co-prevalentes para cada 10 mil habitantes. A investigação insuficiente de $\operatorname{contatos}^{42}$, a centralização das atividades de controle e o envolvimento de poucos profissionais, entre outros, contribuem com a perda de oportunidade de diagnóstico precoce.

Conclui-se que a probabilidade de um indivíduo com hanseníase apresentar lesões de hanseníase em áreas visíveis independe de sexo e idade e essas se manifestam principalmente nos braços. A ocorrência dessas lesões foi verificada em maior freqüência nos indivíduos multibacilares, assim como naqueles com incapacidade física localizada na região ocular e dos membros superiores.

\section{Referência}

1. Ministério da Saúde. Secretaria de Vigilância em Saúde. Sistema Nacional de Vigilância em Saúde. Relatório de Situação. Mato Grosso. 2a ed.Brasília, DF; 2006.
2. Ignotti E. "Abandonos ou Abandonados" [dissertação de mestrado] Rio de Janeiro: Escola Nacional de Saúde Pública - Fundação Osvaldo Cruz; 1999. 
3. Ignotti E, Andrade VLG, Sabrosa PC, Araujo, A.J.G. Estudo da Adesão ao Tratamento da Hanseníase no Município de Duque de Caxias. Hansenol Int 2001;26(1): 23-30.

4. World Health Organization. Global strategy for further reducing the leprosy burden and sustaining leprosy control activities 2006-2010. Operational guidelines. Regional office for South-East Asia. New Delhi; 2006.

5. International Leprosy Association. Relatório do Fórum técnico da ILA. Resumo. Paris; 2002. Disponível em: http://www.google.com.br/search?hl=pt$\mathrm{BR} \& \mathrm{q}=$ relatorio+do+forum+tecnico + da+ila\&meta $=$. [Acessado em 15 de agosto de 2007].

6. Ministério da Saúde. Secretaria de Políticas Públicas. Departamento de Atenção Básica. Área Técnica de Dermatologia Sanitária. Hanseníase: Atividades de Controle e Manual de Procedimentos. Brasília, DF; 2001.

7. Ponnighaus JM, Fine PE, Gruer PJ, Maine N. The anatomical distribution of single leprosy lesions in an African population, and its implications for the pathogenesis of leprosy. Lepr Rev 1990; 61(3): 242-50.

8. Avelleira JCR, Viana FR, Coutinho RBGA, Boechat AM, Andrade VLG. Localização das lesões únicas em pacientes hansenianos. An Bras Dermatol 1992; 67(5):261-3.

9. Katoch K, Natrajan M, Yadan VS, Bhatia AS. Response of Leprosy patients with single lesion to MQT. Acta Leprol 1995; 9(3):133-7.

10. Abraham S, Monzhin NM, Joseph GA, Kurian N, Rao PS, Job CK. Epidemiological significance of first skin lesion in leprosy. Int J Lepr Other Mycobac Dis 1998; 66(2):131-9.

11. Las Aguas JT de. Classificación y formas clínicas. In: La Lepra pasado, presente y futuro. Generalitat Valenciana 1999. p. 119-23.

12. Costa LCV, Andrade KLC, Carmo MAV, Ferreira MAA, Garrocho AA. Manifestações Bucofaciais da Hanseníase. Rev CROMG 2002; 8(3): 191-7.

13. Almeida CAP, Zimmermann RD, Cerveira JGV, Julivaldo FSN. Prontuário Odontológico - Uma orientação para o cumprimento da exigência contida no inciso VIII do art. $5^{\circ}$ do Código de Ética Odontológica. Relatório final apresentado ao Conselho Federal de Odontologia. Rio de Janeiro; 2004.

14. Conselho Federal de Odontologia. Código de Ética Odontológica. Rio de Janeiro, RJ; 2006.

15. Instituto Brasileiro de Geografia e Estatística. Censos demográficos e estimativas. 2007. Disponível em http:// tabnet.datasus.gov.br/cgi/tabcgi.exe?ibge/cnv/popmt. def. [Acessado em 30 de janeiro de 2007.]

16. Mattos RA. Os sentidos da integralidade: algumas reflexões acerca de valores que devem ser defendidos. In: Pinheiro R, Mattos RA, org. Os sentidos da integralidade na atenção e no cuidado à saúde. Rio de Janeiro: IMS-UERJ; 2001. p. 39-64.
17. Aerts D, Abegg C, Cesa K. O papel do cirurgião-dentista no Sistema Único de Saúde. Cien Saude Colet 2004; 9(1): 131-8.

18. Krishnamurthy P. Diagnosis of leprosy. J Indian Med Assoc 2006; 104(12): 680-5.

19. Kumar RB et al. Gender differences in epidemiological factors associated with treatment completion status of leprosy patients in the most hyperendemic district of Nepal. Southeast Asian J Trop Med Public Health 2004; 35(2): 334-9.

20. Kerr-Pontes LR, Barreto ML, Evangelista CM, Rodrigues LC, Heukelbach J, Feldmeier H. Socioeconomic, environmental, and behavioural risk factors for leprosy in Northeast Brazil: results of a case-control study. Int J Epidemiol 2006; 35(4): 994-1000.

21. Sehgal VN, Sehgal S. Leprosy and young urban children. Int J Dermatol 1988; 27(2): 112-4.

22. Cestari TF. Hanseníase na infância. Estudo epidemiológico e clínico - evolutivo dos casos ocorridos em menores de 8 anos no Estado de Rio Grande do Sul no período de 1940 a 1988 [tese de mestrado]. Rio de Janeiro: Universidade Federal do Rio de Janeiro;1990.

23. Sehgal VN, Chaudhry AK. Leprosy in children: a prospective study. Int J Dermatol 1993; 32(3): 194-7.

24. Ministério da Saúde. Secretaria de Vigilância em Saúde. Departamento de Vigilância Epidemiológica. Programa Nacional de Eliminação da Hanseníase. Programa Nacional de Eliminação da Hanseníase em nível municipal 2006-2010. Brasília, DF; 2006.

25. Silva Souza C, Bacha JT. Delayed diagnosis of leprosy and the potential role of educational activities in Brazil. Lepr Rev 2003; 74(3): 249-58.

26. Meima A, Saunderson PR, Gebre S, Desta K, van Oortmarssen GJ, Habbema JD. Factors associated with impairments in new leprosy patients: the AMFES cohort. Lepr Rev 1999; 70(2): 189-203.

27. Rojas V de, Hernández O, Gil R. Some factors influencing delay in leprosy diagnosis. Bull Pan American Health Organization 1994; 28(2): 156-62.

28. Opromolla DVA, Opromolla MA, Ura S. Manutenção da endemia hansênica. Hansenol Int 2003; 28(1): 85-8.

29. Cakiner T, Yüksel A, Soydan M, Saylan T, Bahçeci E. Women and leprosy in Turkey. Indian J Lepr 1993; 65(1): 59-67.

30. Vlassoff C, Khot S, Rao S. Double jeopardy: women and leprosy in India. World Health Stat Q 1996; 49(2): 120-6.

31. Peters ES, Eshiet AL. Male-female (sex) differences in leprosy patients in southeastern Nigeria: females present late for diagnosis and treatment and have higher rates of deformity. Lepr Rev 2002; 73(3): 262-7. 
32. Al-Kandari S, al-Anezi A, Pugh RN, al-Qasaf F, al-Abyad S. Leprosy in Kuwait: an epidemiological study of new cases. Ann Trop Med Parasitol 1990; 84(5): 513-22.

33. Morrisson A. A woman with leprosy is in double jeopardy. Lep Rev 2000; 71(2): 128-43.

34. Alfieri $\mathrm{N}$ et al. Oral lesions in borderline and reactional tuberculoid leprosy. Oral Surg Oral Med Oral Pathol 1983; 55(1): 52-7.

35. Opromolla DVA, Opromolla MA, Ura S. Lesões dimorfas na cavidade oral. Hansenol Int 2003; 28(2); 151-5.

36. De Abreu MAMM, Michalany NS, Weckx LLM, Pimentel DRN, Hirata CHW, Alchorne MMA. A mucosa oral na hanseníase: um estudo clínico e histopatológico. Rev Bras Otorrinolaringol 2006; 72(3): 312-6.

37. Andrade V, Moreira T, Soares RCFR. Impacto da descentralização do Programa de Eliminação da Hanseníase no Brasil. In: Yadon ZE; Gürtler RE; Tobar F; Medici AC (ed.). Descentralización y gestion del control de Las Enfermedades Transmisibles en América Latina. Buenos Aires, Argentina, 2006, p. 165-185.

38. Ministério da Saúde. Secretaria de Vigilância em Saúde. Coordenação Nacional do Programa de Eliminação da Hanseníase. Auto-instrução em análise de situação da endemia de Hanseníase. Brasília, DF; 2004.
39. Ministério da Saúde. Secretaria de Vigilância em Saúde. Programa Nacional de Eliminação da Hanseníase. Cartas de Eliminação da Hanseníase por estado (2a edição) Disponível em: http://portal.saúde.gov.br/ portal/svs. [Acessado em 1 de outubro de 2005.]

40. Lana FCF, Amaral EP, Franco MS, Lanza FM. Detecção da hanseníase no vale de Jequitinhonha - Minas Gerais: redução da tendência epidemiológica ou problemas operacionais para o diagnóstico? Hansenol Int 2004; 29(2): 118-23.

41. Oliveira BFA, Ignotti E, Hartwuig SV, Scatena JH, Andrade VLG. Acréscimo na detecção de casos de hanseníase como resultado da vigilância de comunicantes em 15 municípios considerados prioritários do estado do Mato Grosso, Brasil. Rev Espaço Para a Saúde [periódico na internet]. 2007; 8(2): 11-9. Disponível em http://www.ccs.uel.br/espacoparasaude. [Acesso em 15 de agosto de 2007.]

42. Ignotti E, Bayona M, Alvarez-Garriga C, Andrade VL, Valente JG. Transmission of Hansen's disease and unscreened household contacts. Indian J Lepr 2007; 79(1): 11-25.

Recebido em: 02/06/08

Versão final reapresentada em: 30/09/08 Aprovado em: 17/10/08 